\title{
Obituary for Prof. Roberto Passariello
}

\author{
Carlo Catalano
}

Published online: 24 April 2014

(C) European Society of Radiology 2014

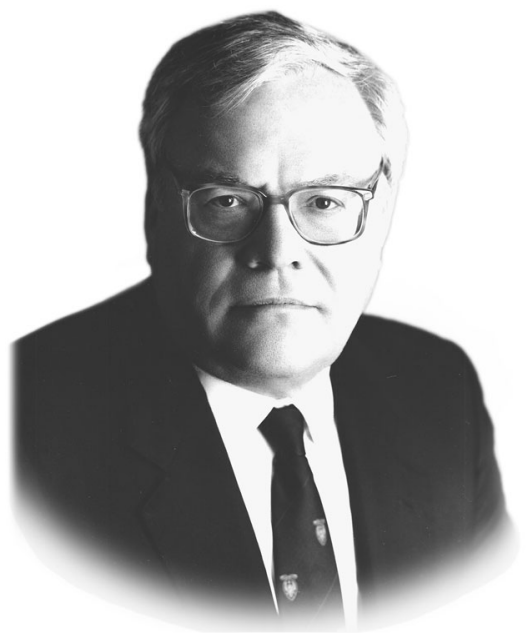

Prof. Roberto Passariello, one of the most respected and wellrecognized leaders in European radiology, suddenly died at the age of 72 in his home in Rome on 11 August 2013.

Prof. Passariello was born in Rome on 23 January 1941. After graduating from high school he began his studies in medicine, which he successfully completed in 1965.

During his studies, he was a brilliant swimmer and waterpolo player. At the age of 18 , he broke the record for crossing the Strait of Sicily, crossing $5.2 \mathrm{~km}$ in open water in 52 minutes and 25 seconds, a record which remained undefeated for 13 years. He was a national major league water-polo player and, although very young, in 1960 he participated in the selections for the national team, which that same year won the gold medal at the Olympic Games.

\section{Catalano $(\square)$}

Department of Diagnostic Radiology, La Sapienza University Hospital, Rome, Italy

e-mail: Carlo.Catalano@uniroma1.it
After completing his residency in radiology in Padua in 1967, he became an assistant at the University of Perugia, where he worked until 1970.

In 1970, he moved to Policlinico Umberto I in Rome, where he commenced interventional radiology in Italy, together with Prof. Plinio Rossi and Prof. Giovanni Simonetti. At that time he started cultural exchanges with colleagues worldwide and the process of internationalization of Italian radiology. He then organized the CARVAT meeting which has remained an annual Roman appointment for 20 years, being attended by over 2,000 radiologists from all over the world.

In 1977 he was recruited by the University of L'Aquila to become chairman of the radiology department; shortly thereafter he obtained a grant to install and develop the first MR device in Italy, a 0.5 -T prototype. Very rapidly L'Aquila, a small town in central Italy and home of a newborn university, became a reference site for Italian and European radiologists willing to comprehend and study this new modality with all its potential. While in L'Aquila, Prof. Passariello and his team of young pupils explored several potential applications of MR imaging, such as neuro, cardiac, abdominal, musculoskeletal and vascular. The first meeting of the MR Angio club (of which he was a co-founder), currently at its 26th edition, was held in L'Aquila in 1989, when MRA was a real challenge.

In L'Aquila Prof. Passariello funded and started the European Seminars of Diagnostic and Interventional Radiology (ESDIR), opening the way to cultural exchanges between western and eastern European countries. At the beginning of the 1990s, as coordinator of the European grant TEMPUS, he worked hard for the diffusion of radiology culture in eastern European countries and for the integration of eastern European radiologists within the European Association of Radiology (EAR) and more recently the European Society of Radiology (ESR).

In 1991 Prof. Passariello was recruited by the University of Rome "La Sapienza" as chairman of the 
radiology department, which had encountered several years of decline. He very rapidly transmitted to his co-workers enthusiasm and passion and made that same department one of the most advanced and well-known throughout Europe.

His involvement in the growing field of radiology in Europe became ever more intense and he became President of ECR in 1999 and of the EAR immediately after that.

Professor Passariello was a prolific researcher, authoring and co-authoring more than 900 scientific articles and 22 books. His book on technology in radiology, of which several editions have been published, will be long be remembered as a milestone for all interested in technological aspects applied to diagnostic imaging. For his competence and visionary ideas he has been greatly appreciated by industry; dedicated MR systems for imaging of the extremities resulted from his thoughts.
Professor Passariello received many honorary memberships, such as that of the Radiological Society of North America (RSNA), and gold medals, such as the ESR Gold Medal. Above all, he has always been esteemed, appreciated and respected by all radiologists and personnel that were lucky enough to work with him. He will be remembered as an enthusiastic leader and recognized as such in Italy, Europe and worldwide, by all of them and especially by his pupils, many of whom have achieved, under his guidance, major roles in radiology.

He was an enjoyable and pleasant gentleman, having friends all over the world and being respected for his wisdom, culture, knowledge, and integrity.

$\mathrm{He}$ is survived by his wife Pauline, his children Maurizio and Luca, and his three grandchildren. 